\title{
FACTORES DE LA ORGANIZACIÓN DEL MERCADO DE LAS PLANTAS MEDICINALES EN IQUITOS - AMAZONÍA PERUANA
}

\author{
Sébastien Galy ${ }^{1}$, Elsa Rengifo ${ }^{2}$ y Yann Olivier Hay ${ }^{1}$
}

\section{RESUMEN}

La ciudad de Iquitos, conocida por muchos como la capital de la Amazonía Peruana, es el lugar donde se comercializa en grandes volúmenes y con una antigüedad de casi cuatro décadas, una gran variedad de especies amazónicas de uso medicinal (Dourojeanni, 1990).

El creciente comercio de plantas medicinales, ocasionado por la demanda de los mercados locales, nacionales e internacionales, para el abastecimiento de diferentes clientes -laboratorios de la industria de fitomedicamentos, universidades e institutos de investigación, entre los principales- es una de las principales causas del interés y explotación selectiva de dichas especies.

La utilización por la población de aproximadamente 500 plantas medicinales, $134 \mathrm{de}$ ellas en forma comercial en el Pasaje Paquito de Iquitos, contribuye -en forma destacada- al cuidado de la salud y a la generación de ingresos económicos en el poblador amazónico. De estas plantas, solo 19 tienen un alto valor comercial.

El potencial de la comercialización de plantas medicinales es todavía muy grande, pero el mal manejo actual de estos recursos naturales nos puede llevar a una situación crítica.

Palabras clave: Plantas medicinales, mercados, recursos naturales, potencial económico.

1 Ingenieros de desarrollo rural en países tropicales, Médecins Aux Pieds Nus. 222 rue Vaugirard 75015 Paris - France. Tele-fax : 00331456707 33. E-mail: www.mapn.org

2 Bióloga, responsable del Proyecto Plantas Medicinales y Biocidas de la Amazonía Peruana, Programa de Aprovechamiento Sostenible de la Biodiverdidad. Instituto de Investigaciones de la Amazonía Peruana. Avda. A. Quiñones km 2,5. Iquitos (Perú). 


\section{RÉSUMÉ}

La ville d'Iquitos, capital de l'Amazonie péruvienne, est un lieu important de commerce de plantes médicinales, et ce depuis presque 4 décenies (Dourojeanni, 1990).

La demande croissante en plantes médicinales, sous l'influence des marchés locaux, nationaux et internationaux, pour l'approvisionnement de différents clients comme les laboratoires, universités et instituts de recherches, est une des principales causes de l'intérêt et de l'exploitation sélective des espèces concernées.

La population d'Iquitos utilise une moyenne de 500 plantes de façon traditionelle, dont 134 d'entre elles sont commercialisées au "Pasaje Paquito" d'Iquitos, ce qui contribue, de manière parallèle, à l'amélioration de la santé et la création d'un micromarché local. De toutes ces plantes, seules 19 ont une haute valeur commerciale.

Le potentiel de la commercialisation des plantes médicinales est encore très important, mais la gestion actuelle des ressources naturelles pourrait nous mener très vite à une situation critique.

Mots Clés: Plantes médicinales, marché, ressources naturelles, potentiel économique.

\section{ABSTRACT}

The city of Iquitos, the capital of Peruvian Amazon, is the place where an important amount of various medicinal plants is being exchanged since 4 decades ago (Dourojeanni, 1990).

The growing trading of medicinal plants, due to the demand of local, national and international markets for the supply of different clients like laboratories, universities and research institutes is one of the main causes of the interest and the selective exploitation of these species.

The inhabitants of Iquitos use approximately 500 medicinal species, from whom 134 are sold in the "Pasaje Paquito". This contributes to health and economy of a part of the Amazonian population. Of these plants, 19 have high commercial value.The potential of the commercialization of medicinal plants is still very important, but the present management of these natural resources can lead to a critical situation. 
Key words: Medicinal plants, markets, natural resources, economic potential.

\section{INTRODUCCIÓN}

La comercialización de algunos recursos naturales, entre ellos las plantas medicinales económicamente claves para empresas farmacéuticas y químicas a escala mayor, es un potencial con que cuenta la Amazonía. Este recurso, con un buen manejo y leyes apropiadas, puede generar divisas y mejorar la economía local y nacional (PNUD, 1997). Asimismo, frente a los costosos medicamentos manufacturados, es una alternativa para la cura y prevención de enfermedades en las personas de bajos recursos económicos y para las que prefieren la opción de lo natural.

Sin embargo, esta actividad comercial es difícil de evaluar, pues se caracteriza por la escasez de datos confiables (Padoch, 1992).

Para dar cuenta de lo que acontece con la comercialización de la plantas medicinales amazónicas, presentamos la experiencia del Pasaje Paquito en el mercado de Belén y la experiencia de las empresas ubicadas en Iquitos que comercializan estos recursos a nivel nacional e internacional.

Ad portas del siglo XXI, realizamos un estudio preliminar que, aunque no nos permita alcanzar conclusiones definitivas, sí nos permite formular algunas hipótesis que deberán ser confirmadas por investigaciones más largas en el futuro.

\section{MATERIALES Y MÉTODOS}

Este estudio se realizó en la ciudad de Iquitos, capital de Loreto, durante los meses de marzo a junio de 1999, como parte de las actividades del convenio entre el Instituto de Investigaciones de la Amazonía Peruana -IIAP- y la ONG francesa Médecins Aux Pieds Nus (Médicos Descalzos).

Se seleccionó esta ciudad porque abriga un mercado local de venta de plantas medicinales muy desarrollado y por ser uno de los lugares donde se ubican siete empresas que comercializan plantas medicinales en el ámbito nacional e internacional (Galy, Rengifo, Hay, 1999).

El trabajo consistió en realizar entrevistas y encuestas sobre los temas de comercialización, extracción y cultivo, a los vendedores de plantas medicinales y a perso- 
nas que tenían vínculos con el negocio; asimismo, se visitó y rescató información en instituciones públicas y privadas. A la vez, se realizó una búsqueda de información a través de Internet.

Se visitó un total de 70 personas, a 45 de las cuales se les aplicó encuestas estructuradas.

\section{RESULTADOS}

\subsection{Mercado local}

El mercado de Belén se ubica en el extremo sur de la ciudad de Iquitos, en la orilla del río Itaya, escenario que ha sufrido cambios en el transcurrir del tiempo. El mercadillo de plantas medicinales se inició aquí a finales de la década de los 60 con las señoras María de Marreros y María de Yap; luego, el número de personas dedicadas a este negocio se fue incrementando paulatinamente.

En 1994 existían 28 puestos de venta, en su mayoría bajo la responsabilidad de una mujer. Podríamos decir que esta actividad de comercialización es un espacio donde las mujeres pueden desarrollar y generar ingresos económicos. En 1999, existían 32 puestos en esta zona y, además de éstos, una ampliación de 10 puestos adicionales donde las productoras vendían directamente sus productos. Desde 1997 se han establecido siete puestos permanentes atendidos por mujeres; la mayoría de ellas se ha especializado en plantas frescas. En los mercados Modelo y Central, se observa también la venta, en pequeña escala, de plantas medicinales.

El total de especies comercializadas en el mercado de Belén es de 134 (Cuadro 1).

El proceso se inicia con el extractor para la mayoría de las especies, quien vende la materia prima al rematista y éste al comerciante, en algunos casos al acopiador, siguiendo una secuencia en el flujo de la comercialización (Figura 2). El valor final de una especie depende de varios factores como disponibilidad de la especie, lugar de acopio y demanda, entre los principales. Estas plantas, en su mayoría, no provienen de áreas de cultivo, sino de bosques naturales con el 60,4 \% (Figura 3). Según sus formas de comercialización, hemos distinguido 10 categorías (Figura 4). Las que ocupan el primer lugar son las plantas en estado fresco, con una variedad de 62 especies; en segundo lugar las cortezas, con 27 especies; las raíces, con 22 especies; 
licores a base de corteza, hojas, raíces solas y/o combinadas, con 19 variedades y, finalmente, harinas /polvos 12; resinas 8; frutos 5; semillas 4; aceites 4 y flores 4 .

\subsection{Empresas}

De las siete empresas que comercializan plantas medicinales en Iquitos (Cuadro 2), sólo cinco tienen su sede aquí; las otras dos tienen sólo una sucursal en esta ciudad. La más antigua tiene 10 años, lo que demuestra que este comercio está todavía joven en la región Loreto.

Cinco de las empresas tienen una relación con un laboratorio de investigación, porque eso les beneficia en varias formas: primero, los laboratorios de investigación pueden ser clientes estables e importantes cuando están haciendo estudios de una planta. En segundo lugar, esta cooperación es un indicador de seriedad y permite validar científicamente los productos comercializados. Las empresas que no tienen este tipo de convenio recurren a investigadores individuales para certificar la buena cualidad de los productos.

\subsection{Plantas con alto valor comercial}

Todavía es difícil evaluar de manera precisa la cantidad de especies medicinales del bosque amazónico. De las 60000 a 90000 especies vegetales estimadas en la región, solamente entre 2000 y 3000 especies fueron inventariadas en la farmacopea y, aproximadamente, 500 de ellas son utilizadas en la ciudad de Iquitos (Mejia, Rengifo, 1997).

Para que sea comercializada a nivel nacional o internacional, una planta debe haber sido estudiada por institutos de investigación para mostrar el efecto terapéutico y la ausencia de toxicidad. Por estas razones, el número de plantas con alto valor comercial es reducido en comparación con las que son usadas por la población local. La lista de estas plantas comercializadas a nivel nacional e internacional asciende a 19 especies (Cuadro 3).

De las 19 especies vegetales comercializadas, sólo 6 se venden en gran cantidad (Cuadro 4). Las otras representan ventas puntuales, de pequeñas cantidades, y muy irregulares. Eso explica las variaciones importantes notadas entre las ventas del año 1998 y las de los cinco primeros meses de 1999. 
La inestabilidad vinculada con este comercio es un fenómeno que concierne a la totalidad de las especies, hasta las especies mundialmente conocidas como la 'uña de gato' (Uncaria sp.) o el 'ayahuasca' (Banisteriopsis caapi). En tal sentido, es muy difícil anticipar la evolución del mercado, tanto por los políticos y los investigadores como por los empresarios mismos.

\subsection{Destinos de los productos}

Si bien existe un mercado nacional, los empresarios no tienen una explicación del motivo por el cual se satura rápidamente.

La gran mayoría de las plantas medicinales tiene como destino la ciudad de Lima. Sin embargo, una pequeña parte de productos es exportado directamente desde Iquitos hacia Europa, Japón o Estados Unidos.

El fenómeno de convergencia de productos en Lima se explica por el hecho que la mayoría de las plantas, vendidas como materia primaria por las empresas de Iquitos, es comprada por empresas de Lima que se encargan de su procesamiento en cápsulas u otras formas de presentación. Estos productos son luego exportados hacia los países extranjeros, sobre todo los occidentales.

Solamente una empresa loretana tiene las máquinas necesarias para procesar las plantas, lo que ilustra bien el retraso de la región Loreto en comparación con la ciudad de Lima, donde está concentrada la casi totalidad de la industria y la tecnología.

\subsection{Extracción del bosque}

No hemos podido determinar de manera precisa ni las características ni los lugares de extracción de las especies medicinales del bosque, porque las grandes distancias y las limitaciones logísticas existentes en la Amazonía Peruana no facilitan este trabajo. En todo caso, podría ser el tema de un programa de investigación más largo. Sin embargo, se nota que hay algunas zonas más apropiadas para la extracción por las facilidades de transporte hacia Iquitos. Las tres zonas de extracción más importantes son el río Napo, el río Nanay y la carretera Iquitos-Nauta.

El extractivismo sigue modelos de funcionamiento muy específicos. Entre las empresas encuestadas en Iquitos, se nota tres tipos de extractivismo: 
- Algunas empresas trabajan con extractores, quienes están empleados a tiempo completo en la cosecha y el transporte de las plantas medicinales hasta la ciudad de Iquitos. Esta mano de obra es calificada y tiene capacidades en determinación botánica de las especies vegetales. Es frecuente encontrar varias especies con un sólo nombre vernacular (por ejemplo, 'uña de gato' corresponde a $U$. tomentosa y $U$. guianensis) y esta confusión es un obstáculo que impide a las empresas trabajar con las poblaciones locales.

- Otras empresas trabajan con extractores ocasionales. Estas personas, que son las que sacan y transportan las plantas, son empleadas solamente en caso de existir pedidos. Esta mano de obra es también calificada y sabe evitar la confusión referida en el caso anterior.

- Por último, otras empresas proceden de una tercera manera para abastecerse. En caso de pedido, se van a las comunidades para comprar directamente las especies necesitadas en la cantidad requerida. El empresario hace una supervisión del trabajo para verificar que se extrae la especie correcta. Este modo de operación se practica particularmente con las comunidades nativas que son propietarias de territorios de gran superficie y riqueza vegetal.

Eventualmente, una empresa puede abastecer a otra empresa en caso de pedidos puntuales y en pequeñas cantidades. Este caso se da generalmente con especies raramente comercializadas, cuyo costo de prospección y extracción es más alto que el de la compra directa del producto a otra empresa.

\subsection{Cultivo de especies medicinales}

La ausencia de informaciones fiables nos impide cuantificar el recurso de cada planta con alto valor comercial, lo que debería animar a recurrir a un cierto principio de precaución. El cultivo es la forma más segura de garantizar la perennidad de las especies y, por ende, del mercado. La legislación actual incentiva el cultivo de las especies medicinales que tienen un alto valor comercial. Sin embargo, algunos obstáculos hacen que el cultivo de las plantas medicinales no sea muy practicado por el momento.

De todas las empresas encuestadas, una sola practica el cultivo de la especie que comercializa y éste ha sido impuesto por el laboratorio cliente. Se trata de 5000 árboles de sangre de grado (Croton lechleri) que fueron sembrados en 1992 y que van a poder ser cosechados a partir del año 2000. Como el ayahuasca (Banisteriopsis 
caapi) y la uña de gato (Uncaria sp.), la sangre de grado fue investigada suficientemente para asegurar un mínimo de datos fiables sobre su cultivo.

En lo que respecta a las otras seis empresas, se disciernen dos tipos de reacción:

- Cuatro de ellas no tienen previsto cultivar las plantas medicinales por razones económicas. El cuidado del terreno, la mano de obra necesaria y todos los otros gastos representan una inversión elevada, sobre todo cuando la extracción del medio silvestre es totalmente gratuita. De otro lado, la inestabilidad actual del mercado de la mayoría de las especies comercializadas no permite evaluar precisamente el tipo y la cantidad de especies a cultivar, existiendo siempre el riesgo de que desaparezca el mercado para un producto determinado.

- Desde hace algún tiempo, dos empresas están empezando a invertir en el cultivo $\mathrm{y}$ en viveros de plantas medicinales. Pero las características de este cultivo varían según las especies. Para la sangre de grado (Croton lechleri) se practica un cultivo de unas centenas de plantones; para la chacruna (Psychotria viridis), el mapacho (Nicotiana tabacum) y el jergón sacha (Dracontium loretense), se hace un cultivo de unas decenas de plantones. Eso se debe, sea a un pedido mínimo (jergón sacha), sea a una ausencia de datos agronómicos que desanima a los empresarios a cultivar en mayor escala. En este caso, el cultivo que se está realizando se parece más a un "ensayo agronómico" que a un verdadero cultivo. Para la uña de gato (Uncaria sp.) y el ayahuasca (Banisteriopsis caapi), a pesar de los datos agronómicos accesibles, no se ha empezado en Loreto un cultivo significativo para satisfacer la demanda del mercado internacional.

\section{DISCUSIÓN}

\subsection{Peligros de la explotación de especies medicinales}

\section{Problema de la extracción}

A pesar de las advertencias de los científicos sobre la importancia de una gestión inteligente del recurso vegetal amazónico, la extracción directa del bosque es por el momento la fuente más frecuente de acopio por parte de las empresas, porque es la más simple y rentable a corto tiempo. Las empresas que encuentran dificultades para emprender el cultivo de especies medicinales en gran escala, afirman que sin "un 
apoyo efectivo del gobierno", nunca el cultivo podrá ser utilizado como alternativa de producción frente a la extracción.

La inestabilidad del mercado internacional, la falta de datos agronómicos y de información relativa a los costos de producción no incentivan el cultivo para la mayoría de las plantas con alto valor comercial. Pero para la uña de gato, que tiene un mercado asegurado y datos agronómicos y económicos, no se está realizando un plan de cultivo para lograr la sostenibilidad del recurso. Igualmente, nos podemos preguntar si se cultivaría la sangre de grado sin la presión actual que manifiesta el laboratorio cliente.

En estas condiciones, todos los interesados deben tener conciencia de su función y su interés respectivo en el manejo sostenible de plantas medicinales. A nivel político, es fundamental que el apoyo del gobierno no sólo se reduzca a la simple asignación de terrenos, que frecuentemente no son utilizados, e incluso son desconocidos para algunos propietarios. El gobierno podría incentivar, a través de apoyos especiales, a los empresarios para que presenten un plan de manejo sostenible pactado con los extractores. Sería responsabilidad de los investigadores supervisar estos planes y capacitar a los empresarios y a los extractores, brindando más informaciones agronómicas y económicas e investigando un mayor número de especies de plantas medicinales. Por otro lado, clientes extranjeros no tendrían que seguir con un negocio "ciego" sino, más bien, asegurarse de que los productos comprados no sean extraídos del bosque sin un plan de manejo adecuado.

\section{Peligro de desaparición de las plantas medicinales}

Para poder medir los riesgos que implica la explotación del recurso, es importante verificar cuál es la parte de la planta que se usa, así como el tipo botánico (árbol, arbusto, liana, hierba) y las características de la regeneración de la planta. En la Figura 1 se ilustra el peligro que representa la explotación de las especies medicinales con alto valor comercial. 
Hoja (4) y bulbo (1) Corteza (7)

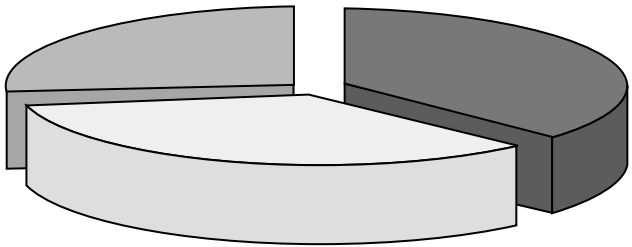

Resina (6) y extracto (1)

Peligroso

Potencialmente

Peligroso

No peligroso

Figura 1: Peligro de explotación de las 19 especies comercializadas.

Los números entre paréntesis se refieren al número de especies.

El peligro es máximo cuando se usa la raíz o la corteza de un especie medicinal que no tiene un crecimiento rápido. En este caso, una sobre-explotación puede llevar rápidamente a la desaparición de la especie, como lo que ha ocurrido con el palo de rosa (Tabebuna rosea). El caso presente del jergón sacha (Dracontium loretense) no representa un peligro por la alta regeneración de esta planta. Respecto a la explotación de las resinas, se considera como potencialmente peligrosa: aunque se pueda sacar la resina sin matar al árbol, la cantidad extraída es mayor cuando se corta el árbol. En este sentido, podría ser interesante ver cuál es la proporción de los que usan cada opción.

La comercialización de hojas o de raíces de hierbas no representa un peligro para la supervivencia de las especies vegetales.

La Figura 1 nos enseña que el futuro de la mayoría de las especies medicinales comercializadas va a depender únicamente del modo de explotación de las poblaciones silvestres, lo que confirma la necesidad de una política nacional de manejo de los recursos medicinales amazónicos. 


\subsection{Evolución del mercado}

La totalidad de los empresarios encuestados están de acuerdo en decir que el futuro de la comercialización de las plantas medicinales es muy prometedor. Sin embargo, el desarrollo de este mercado tendrá consecuencias todavía imprevisibles: la velocidad con la cual se crean las empresas nos induce a preocuparnos por las repercusiones que provocaría, sobre los recursos naturales, un aumento importante de las exportaciones de algunas plantas medicinales.

Si todos los interesados, investigadores, políticos, extractores y comerciantes no prevén esta evolución y no aplican las medidas preventivas pertinentes, los efectos podrían ser nefastos en el corto plazo para la economía y el medio ambiente amazónico.

\section{RECOMENDACIONES}

El mercado de plantas medicinales amazónicas es bastante inestable y no está todavía bien definido para la casi totalidad de especies medicinales, actualmente vendidas en el área de Iquitos.

Una explotación duradera en el tiempo deberá acompañarse de una política adaptada, como podrían ser el cultivo y el manejo adecuado de las especies con una extracción sostenible. De esta actitud depende la sostenibilidad de la cosecha y globalmente el futuro del comercio de las especies vegetales medicinales amazónicas. Podría ser un riesgo para el futuro que esta responsabilidad sea sólo de las comunidades, especialmente nativas, para las cuales la venta de estos productos es una fuente potencial importante de ingresos.

Los cambios de una extracción no controlada del bosque hacia un manejo sostenible de los recursos naturales, se están percibiendo. Pero los varios obstáculos actuales imponen que este plan de manejo sea planteado con todos los protagonistas si se quiere una transición exitosa.

En este delicado contexto, habrá que contar con la conciencia de cada uno: políticos, investigadores, comunidades y empresarios para evitar que este patrimonio sea dilapidado. 


\section{BIBLIOGRAFIA}

DOUROJEANNI, MARC J. 1990. Amazonía ¿que hacer? Iquitos (Perú): Centro de Estudios Teológicos de la Amazonía. 374 pp.

ESTRELLA, E., 1995. Plantas Medicinales Amazónicas: Realidad y Perspectivas. Lima (Perú): Tratado de Cooperación Amazónica.

GALY, S.; RENGIFO, E.; HAY, Y.O. 1999. Estado actual de la comercialización y utilización de plantas medicinales en la región de Iquitos - Amazonía Peruana. Informe interno. IIAP/MAPN. 21 pp.

MEJIA K.; RENGIFO, E. 1997. Plantas medicinales de uso popular en la Amazonía Peruana. Iquitos (Perú): AECI-GRL-IIAP.

PADOCH, C. 1992. Marketing of Non-Timber forest Products in Western Amazonía: General Observations and Research Priorities. In: Advances in Economic Botany 9 : 43-50. The New York Botanical Garden.

PNUD, 1997. Informe sobre el desarrollo humano del Perú, temas y experiencias. New York - EU: Nations Unis. pp. 87-95. 
Cuadro 1: Lista de especies que se comercializan en Iquitos (Pasaje Paquito)

\begin{tabular}{|c|c|c|c|}
\hline Nombre Científico & Nombre Vulgar & Familia & Categoría \\
\hline Abuta grandiflora & Abuta & Menispermaceae & Co \\
\hline Alchornea castaneifolia & Ipururo & Euphorbiaceae & $\mathrm{Fr}, \mathrm{Co}$ \\
\hline Alpiniea nutans & Canelilla* & Zingiberaceae & $\mathrm{Fr}$ \\
\hline Alternanthera braseliana & Lancetilla* & Amaranthaceae & $\mathrm{Fr}$ \\
\hline Anacardium occidentale & Casho $*$ & Anacardiaceae & $\mathrm{Fr}$ \\
\hline Aniba roseadora & Palo de rosa & Lauraceae & Ace \\
\hline Aparisthmium cordatum & Ushaquiro & Euphorbiaceae & $\mathrm{Co}, \mathrm{Li}$ \\
\hline Artocarpus altilis & Pan del arbol & Moraceae & $\mathrm{Re}$ \\
\hline Aspidosperma excelsum & Remocaspi & Apocynaceae & Co \\
\hline Ayapana pallustris & Caguena* & Asteraceae & $\mathrm{Fr}$ \\
\hline Bactris gasipaes & Pijuayo * & Arecaceae & $\mathrm{Ra}$ \\
\hline Banisteriopsis caapi & Ayahuasca & Malpighiaceae & Co \\
\hline Bauhinia glabra & Motelo-sango & Fabaceae & Co \\
\hline Bixa orellana & Achiote $*$ & Bixaceae & Fr,Fru \\
\hline Brosimum acutifolium & Murure & Moraceae & $\mathrm{Re}, \mathrm{Co}$ \\
\hline Brosimum rubescens & Palisangre & Moraceae & $\mathrm{Co}$ \\
\hline Brugmansia aurea & Toé * & Solanaceae & $\mathrm{Fr}$ \\
\hline Brunfelsia grandiflora & Chiric-sanango & Solanaceae & $\mathrm{Ra}, \mathrm{Li}$ \\
\hline Brysonima coriacea & Indano & Malpighiaceae & $\mathrm{Co}, \mathrm{Li}$ \\
\hline Cactus sp. & Chupocasho & Cactaceae & $\mathrm{Fr}$ \\
\hline Cajanus cajan & Puspo poroto $*$ & Fabaceae & $\mathrm{Fr}$ \\
\hline Campsiandra angustifolia & Huacapurana & Fabaceae & $\mathrm{Co}, \mathrm{Li}$ \\
\hline Canna indica & Achira* & Cannaceae & $\mathrm{Se}$ \\
\hline Caryodaphnopsis fosteri & Achuni-sanango & Lauraceae & $\mathrm{Ra}, \mathrm{Li}$ \\
\hline Cassia reticulata & Retama & Fabaceae & Flo \\
\hline Cedrela odorata & Cedro & Meliaceae & Co \\
\hline Cestrum auriculatum & Hierba santa $*$ & Solanaceae & $\mathrm{Fr}$ \\
\hline Chenopodium ambrosoides & Paico * & Chenopodiaceae & $\mathrm{Fr}$ \\
\hline Chlorophora tinctoria & Insira & Moraceae & $\operatorname{Re}$ \\
\hline Cissus sicyoides & Sapo huasca & Vitaceae & Co \\
\hline Citrus limon & Limón * & Rutaceae & $\mathrm{Fr}$ \\
\hline Citrus paradisi & Toronja * & Rutaceae & $\mathrm{Fr}$ \\
\hline Clusia rosea & Renaquilla & Clusiaceae & $\mathrm{Re}, \mathrm{Ra}$ \\
\hline
\end{tabular}




\begin{tabular}{|c|c|c|c|}
\hline Coffea arabica & Café* & Rubiaceae & Fr \\
\hline Coix lacryma-jovi & Rosario & Poaceae & $\mathrm{Se}$ \\
\hline Commelia sp. & Alacrancito* & Commeliacea & $\mathrm{Ra}, \mathrm{Ha}$ \\
\hline Copaifera paupera & Copaiba & Fabaceae & Ace \\
\hline Cornutia odorata & Salvia(Chingura) & Verbenaceae & $\mathrm{Fr}$ \\
\hline Costus arabicus & Caña agria & Zingiberaceae & Fr \\
\hline Couropita guianensis & Ayahuma & Lecythidaceae & Fr \\
\hline Crecentia cujete & Tutumo $*$ & Bignoniaceae & Fru \\
\hline Croton lechleri & Sangre de grado & Euphorbiaceae & $\mathrm{Re}$ \\
\hline Curcuma longa & Guisador * & Zingiberaceae & $\mathrm{Ra}$ \\
\hline Cymbopogon citratus & Yerba Luisa* & Poaceae & Fr \\
\hline Cyperus articulatus & Piri-piri periquito $*$ & Cyperaceae & $\mathrm{Ra}, \mathrm{Ha}$ \\
\hline Cyperus diffusus & Boa piri-piri* & Poaceae & Fr,Ha \\
\hline Desamidium adscenses & Amor seco & Fabaceae & $\mathrm{Fr}$ \\
\hline Dieffenbachia obliqua & Patiquina blanca $*$ & Araceae & $\mathrm{Fr}$ \\
\hline Dioscorea decorticans & Macaquiño & Dioscoreaceae & $\mathrm{Ra}, \mathrm{Ha}$ \\
\hline Dracontium loretense & Jergón sacha & Araceae & $\mathrm{Ra}, \mathrm{Ha}$ \\
\hline Elaeis oleifera & Palma & Arecaceae & Ace \\
\hline Eleuterine bulbosa & Yahuar piri-piri * & Iridaceae & $\mathrm{Ra}$ \\
\hline Erythrina fusca & Amasisa & Fabaceae & Co \\
\hline Erythroxylum coca & Coca $*$ & Erythroxylaceae & Fr \\
\hline Euterpe precatoria & Huasai & Arecaceae & $\mathrm{Ra}$ \\
\hline Fevillea cordifolia & Habilla & Cucurbitaceae & $\mathrm{Se}$ \\
\hline Ficus insipida & Ojé & Moraceae & $\operatorname{Re}$ \\
\hline \begin{tabular}{|l} 
Fittonia verschaffeltii \\
\end{tabular} & Motelillo * & Acanthaceae & $\mathrm{Ha}, \mathrm{Ra}$ \\
\hline Genipa americana & Huito & Rubiaceae & Fru \\
\hline Gossypium barbadense & Algodón* & Malvaceae & $\mathrm{Fr}$ \\
\hline Hibiscus abelmoschus & Mishuishma & Malvaceae & $\mathrm{Fr}$ \\
\hline Himatanthus suucuba & Bellacocaspi & Apocynaceae & $\mathrm{Re}$ \\
\hline Hura crepitans & Catahua & Euphorbiaceae & $\mathrm{Fr}$ \\
\hline Hymenaea oblongifolia & Azucar huayo & Fabaceae & $\mathrm{Co}, \mathrm{Li}$ \\
\hline Jatropa curcas & Piñón blanco * & Euphorbiaceae & $\mathrm{Fr}, \mathrm{Se}$ \\
\hline Jatropa gossypifolia & Piñón rojo $*$ & Euphorbiaceae & $\mathrm{Fr}$ \\
\hline Justicia pectoralis & Cariñito * & Asteraceae & $\mathrm{Fr}$ \\
\hline Kalanchoe pinnata & Hoja del aire & Crassulaceae & $\mathrm{Fr}$ \\
\hline Laportea aestuans & Ishanga colorada & Urticaceae & $\mathrm{Fr}$ \\
\hline Licaria canella & Moena & Lauraceae & Co \\
\hline Lippia alba & Pampa orégano * & Verbenaceae & Fr \\
\hline Ludwigia hyssopifolia & Arco sacha & Onagraceae & $\mathrm{Fr}$ \\
\hline
\end{tabular}




\begin{tabular}{|c|c|c|c|}
\hline Malachra alceifolia & Malva & Malvaceae & $\mathrm{Fr}$ \\
\hline Mansoa alliacea & Ajo sacha hembra * & Bignoniaceae & Fr,Li \\
\hline Mansoa alliacea & Ajo sacha macho* & Bignoniaceae & Fr,Li \\
\hline Maquira coriacea & Capinuri & Moraceae & $\mathrm{Re}$ \\
\hline Maranta arundinacea & Camarón * & Marantaceae & $\mathrm{Ra}, \mathrm{Ha}$ \\
\hline Maranta arundinacea & Shimipampana * & Marantaceae & $\mathrm{Ra}, \mathrm{Ha}$ \\
\hline Maytenus macrocarpa & Chuchuhuasi & Celastraceae & $\mathrm{Co}, \mathrm{Li}$ \\
\hline Menta piperita & Menta * & Labiatae & $\mathrm{Fr}$ \\
\hline Minquartia guianensis & Fierro-caspi & Oleaceae & Co,Li \\
\hline Momordica charantia & Papailla & Cucurbitaceae & $\mathrm{Fr}$ \\
\hline Mucuna rostrata & Ojo de vaca & Fabaceae & $\mathrm{Se}$ \\
\hline Nicotiana tabacum & Tabaco * & Solanaceae & $\mathrm{Fr}$ \\
\hline Ocimun americanum & Sharamasho * & Labiatae & $\mathrm{Fr}$ \\
\hline Ocimun basilicum & Albahaca* & Labiatae & $\mathrm{Fr}$ \\
\hline Ocimun micrantum & Albaquilla* & Labiatae & $\mathrm{Fr}$ \\
\hline Ocotea aciphylla & Canela sacha & Lauraceae & $\mathrm{Co}, \mathrm{Li}$ \\
\hline Oenocarpus bataua & Ungurahui & Arecaceae & Ace \\
\hline Ormosia coccinea & Huayruro & Fabaceae & $\mathrm{Se}$ \\
\hline Passiflora nitida & Granadilla & Passifloraceae & $\mathrm{Fr}$ \\
\hline Passiflora quadrangularis & Tumbo $*$ & Passifloraceae & $\mathrm{Fr}$ \\
\hline Peperomia flavamenta & Congonita & Piperaceae & $\mathrm{Fr}$ \\
\hline Persea americana & Palta * & Lauraceae & $\mathrm{Fr}$ \\
\hline Petiveria alliaceae & Mucura * & Phytolaccaceae & $\mathrm{Fr}$ \\
\hline Phyllantus stipulatus & Chanca piedra & Euphorbiaceae & $\mathrm{Fr}$ \\
\hline Physalis angulata & Bolsa mullaca & Solanaceae & $\mathrm{Fr}$ \\
\hline Piper aduncum & Cordoncillo & Piperaceae & $\mathrm{Fr}$ \\
\hline Piper callosum & Guayusa * & Piperaceae & $\mathrm{Fr}$ \\
\hline Piperpeltata & Santa María & Piperaceae & $\mathrm{Fr}$ \\
\hline Pithyrusa adunca & Suelda con suelda & Loranthaceae & $\mathrm{Fr}$ \\
\hline Plantago major & Llantén * & Plantaginaceae & $\mathrm{Fr}$ \\
\hline Pogostemon heyneanu & Oriza * & Labiaceae & $\mathrm{Fr}$ \\
\hline Polypodium decumanum & Cotochupa & Polypodiaceae & $\mathrm{Ra}$ \\
\hline Portulacca oleraceae & Verdolaga & Portulacaceae & $\mathrm{Fr}$ \\
\hline Pseudobombax munguba & Punga & Bombacaceae & Co \\
\hline Psidium guayaba & Guayaba * & Myrtaceae & $\mathrm{Fr}$ \\
\hline Ricinus comunis & Higuerilla & Euphorbiaceae & $\mathrm{Se}$ \\
\hline Rosa canina & Rosa Castilla * & Rosaceae & Flo \\
\hline Rosa centrifolia & Rosa maceta * & Rosaceae & Flo \\
\hline Sambucus mexicana & Sauco * & Caprifoliaceae & $\mathrm{Fr}$ \\
\hline
\end{tabular}




\begin{tabular}{|llll|}
\hline Schinopsis peruviana & Cocobolo & Anacardiaceae & $\mathrm{Co}, \mathrm{Li}$ \\
\hline Scleria microcarpa & Cortadera & Cyperaceae & $\mathrm{Ra}$ \\
\hline Scoparia dulcis & Nucño-pichana & Scropulariaceae & $\mathrm{Fr}$ \\
\hline Senna aff. bacillaris & Mataro & Fabaceae & $\mathrm{Fru}$ \\
\hline Smilaxfebrifuga & Zarzaparrilla & Smilaceae & $\mathrm{Ra}$ \\
\hline Solanum mammosum & Teta de vaca & Solanaceae & $\mathrm{Fru}$ \\
\hline Spondias mombin & Ubos & Anacardiaceae & $\mathrm{Co}, \mathrm{Li}$ \\
\hline Stachytarpheta cayenesis & Verbena blanca & Verbenaceae & $\mathrm{Fr}$ \\
\hline Swartzia polyphylla & Cumaceba & Fabaceae & $\mathrm{Co}, \mathrm{Li}$ \\
\hline Tabebuia serratifolia & Tahuari & Bignoniaceae & $\mathrm{Co}, \mathrm{Li}$ \\
\hline Tachigalia tessmannii & Tangarana & Fabaceae & $\mathrm{Co}$ \\
\hline Tagetes erecta & Rosa-sisa $*$ & Asteraceae & $\mathrm{Flo}$ \\
\hline Tynnanthus panurensis & Clavo huasca & Bignoniaceae & $\mathrm{Co}, \mathrm{Li}$ \\
\hline Uncaria guianensis & Uña de gato & Rubiaceae & $\mathrm{Co}, \mathrm{Li}$ \\
\hline Uncaria tomentosa & Uña de gato & Rubiaceae & $\mathrm{Co}, \mathrm{Li}$ \\
\hline Unonopsis floribunda & Icoja & Annonaceae & $\mathrm{Co}, \mathrm{Li}$ \\
\hline Verbena officinalis & Verbena negra & Verbenaceae & $\mathrm{Fr}$ \\
\hline Vetiveria zizanoides & Pachuli $*$ & Poaceae & $\mathrm{Ra}$ \\
\hline Xanthosoma heleborifolium & Mano abierta $*$ & Araceae & $\mathrm{Ra}, \mathrm{Ha}$ \\
\hline Xanthosoma sp. & Buzeta hembra* & Araceae & $\mathrm{Ra}, \mathrm{Ha}$ \\
\hline Xanthosoma sp. & Buzeta macho $*$ & Araceae & $\mathrm{Ra}, \mathrm{Ha}$ \\
\hline Xanthosoma violaceum & Patiquina negra $*$ & Araceae & $\mathrm{Fr}$ \\
\hline Zamia ulei & Lengua de perro & Cycadaceae & $\mathrm{Ra}$ \\
\hline
\end{tabular}

\section{Leyenda :}

$*$ = Especies cultivadas

Ace $=$ Aceites

Co $=$ Corteza

Flo $=$ Flores

$\mathrm{Fr}=$ Frescas

Fru $=$ Frutos

Ha $=$ Harinas

$\mathbf{L i}=$ Licores

Ra $=$ Raíces

Re = Resinas

Se $=$ Semillas 
Figura 2: Canales de comercialización de plantas medicinales.

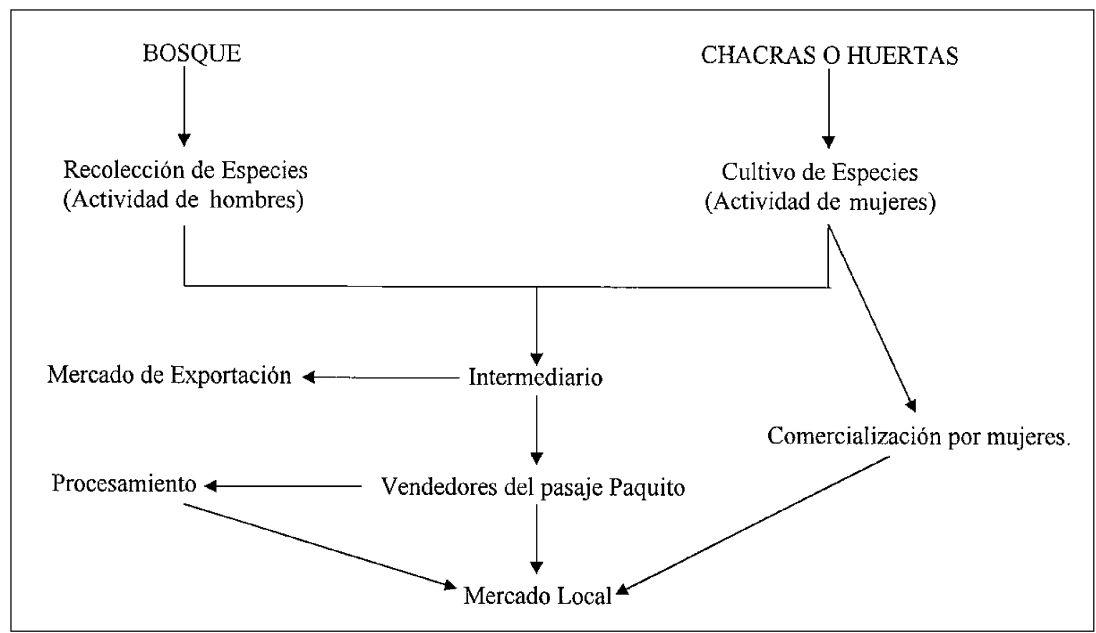

Figura 3: Distribución de especies comercializadas según su procedencia.

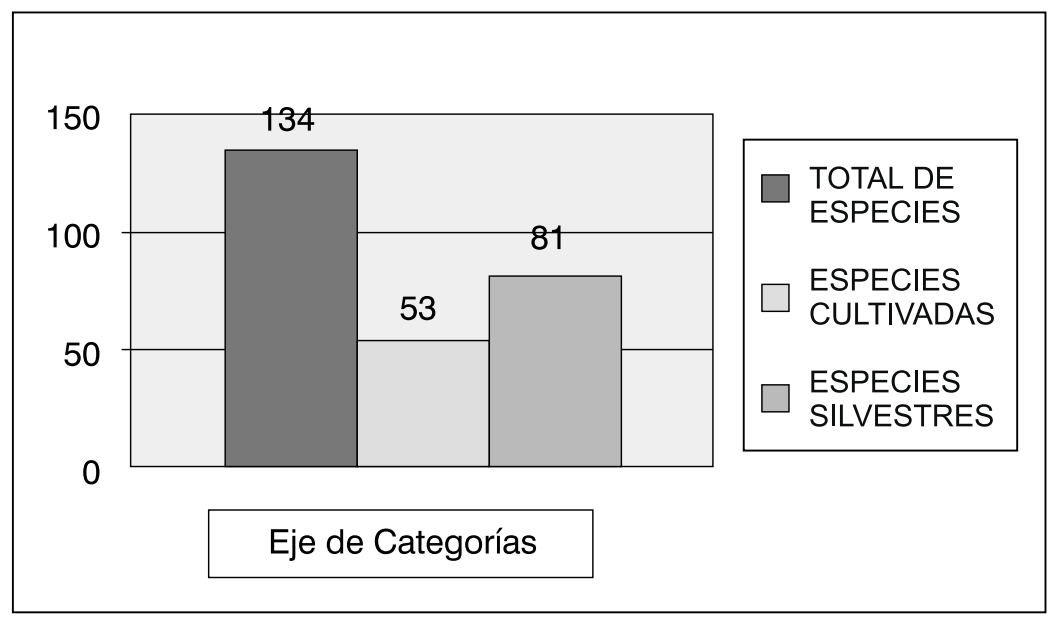


Figura 4: Formas de comercialización de plantas medicinales en el Pasaje Paquito.

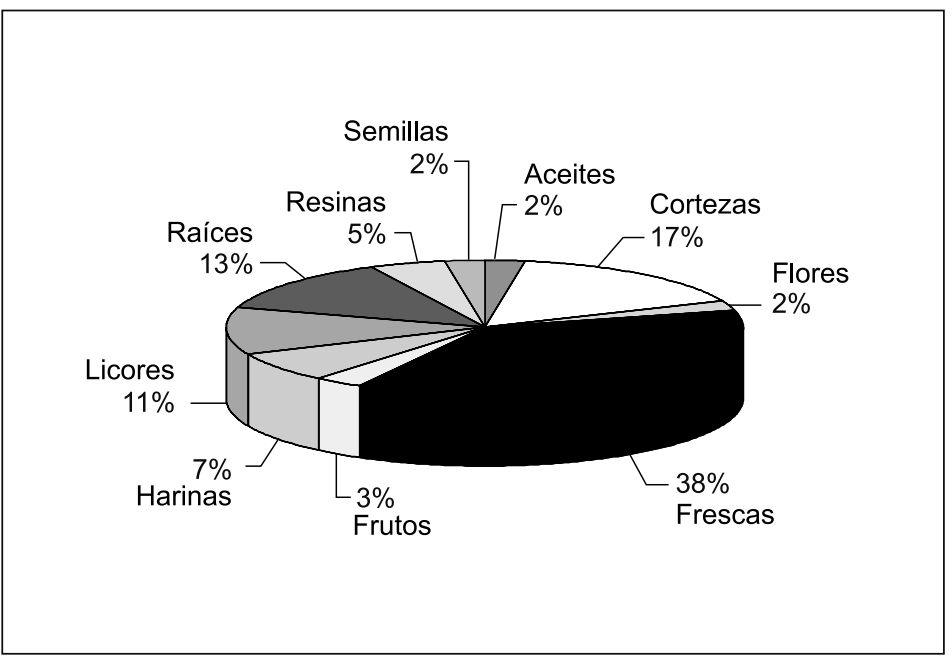

Cuadro 2: Empresas localizadas en Iquitos.

\begin{tabular}{|l|c|c|c|c|c|}
\hline \multicolumn{1}{|c|}{ Nombre } & Origen & $\begin{array}{c}\text { Represen- } \\
\text { tante }\end{array}$ & $\begin{array}{c}\text { Ocupación } \\
\text { anterior }\end{array}$ & Sede & $\begin{array}{c}\text { Relaciones con } \\
\text { laboratorio }\end{array}$ \\
\hline $\begin{array}{l}\text { Servicio Integral de } \\
\text { Comercial }\end{array}$ & 1989 & M.F.P. & Negocio & Lima & $\begin{array}{c}\text { SI (Italo } \\
\text { peruano) }\end{array}$ \\
\hline $\begin{array}{l}\text { Amazonian Natural } \\
\text { Products }\end{array}$ & 1991 & F.A. & Botánico & Iquitos & SI (americano) \\
\hline $\begin{array}{l}\text { Estación Biológica } \\
\text { ISULA }\end{array}$ & 1993 & J.G.C.C. & Médico & Iquitos & SI (canadiense) \\
\hline $\begin{array}{l}\text { Productos Naturales } \\
\text { Amazónicos }\end{array}$ & 1995 & C.G. & Botánico & Iquitos & SI (peruano) \\
\hline R.MUELLA S.A. & 1995 & H.S.G. & Negocio & Lima & SI (peruano) \\
\hline $\begin{array}{l}\text { Chacruna (ex-Semillas } \\
\text { Amazónicas) }\end{array}$ & 1996 & E.B.R. & Agrónoma & Iquitos & NO \\
\hline Laboratorio Selva & 1997 & L.L.V & Químico & Iquitos & NO \\
\hline
\end{tabular}


Cuadro 3: Lista de plantas medicinales exportadas.

\begin{tabular}{|c|c|c|c|c|}
\hline Nombre científico & $\begin{array}{l}\text { Nombre } \\
\text { Vulgar }\end{array}$ & Familia & $\begin{array}{c}\text { Tipo } \\
\text { vegetal }\end{array}$ & $\begin{array}{c}\text { Parte } \\
\text { utilizada }\end{array}$ \\
\hline $\begin{array}{l}\text { Banisteriopsis caapi } \\
\text { (Spruce ex Grise.) }\end{array}$ & Ayahuasca & MALPIGHIACEAE & Liana & Corteza \\
\hline $\begin{array}{l}\text { Brosimum acutifolium } \\
\text { Huber Subsp. }\end{array}$ & Murure & MORACEAE & Arbol & $\begin{array}{l}\text { Corteza } \\
\text { y Resina }\end{array}$ \\
\hline Bursera graveolens (H.B.К.)* & Palo Santo & BURSERACEAE & Arbol & Corteza \\
\hline Copaifera paupera (Herzog) & Copaiba & FABACEAE & Arbol & Resina \\
\hline Croton lechleri Muell.Arg. & $\begin{array}{l}\text { Sangre de } \\
\text { grado }\end{array}$ & EUPHORBIACEAE & Arbol & Resina \\
\hline Dacryodes peruviana (Loes.) & Copal & BURSERACEAE & Arbol & Resina \\
\hline Diplopterys cabrerana & Chariponga & MALPIGHIACEAE & Liana & Hoja \\
\hline $\begin{array}{l}\text { Dracontium loretense } \\
\mathrm{K} . \text { Krause }\end{array}$ & Jergón sacha & ARACEAE & Hierba & Bulbo \\
\hline Ficus insipida (Will.) & Ojé & MORACEAE & Arbol & Resina \\
\hline Genipa americana $\mathrm{L}$. & Huito & RUBIACEAE & Arbol & Extracto \\
\hline $\begin{array}{l}\text { Hevea brasiliensis (Will.) } \\
\text { Muell. Arg. }\end{array}$ & Chiringa & EUPHORBIACEAE & Arbol & Resina \\
\hline $\begin{array}{l}\text { Maytenus macrocarpa } \\
(\mathrm{R} \& \mathrm{P}) \text { Briquet }\end{array}$ & Chuchuhuasi & CELASTRACEAE & Arbol & Corteza \\
\hline Nicotiana tabacum $\mathrm{L}$. & $\begin{array}{c}\text { Mapacho o } \\
\text { Tabaco }\end{array}$ & SOLANACEAE & Hierba & Hoja \\
\hline Psychotria viridis $\mathrm{R} \& \mathrm{P}$ & Chacruna & RUBIACEAE & Liana & Hoja \\
\hline $\begin{array}{l}\text { Trichocereus pachanoi } \\
\text { (Britton \& Rose)* }\end{array}$ & San Pedro & CACTACEAE & Cactus & Hoja \\
\hline $\begin{array}{l}\text { Uncaria tomentosa (Will.) } \\
\text { D.C. }\end{array}$ & Uña de gato & RUBIACEAE & Liana & Corteza \\
\hline $\begin{array}{l}\text { Uncaria guianensis (Aubl.) } \\
\text { Gmelin }\end{array}$ & Uña de gato & RUBIACEAE & Liana & Corteza \\
\hline Virola calophylla Warburg & $\begin{array}{l}\text { Cumala } \\
\text { blanca }\end{array}$ & MYRISTICACEAE & Arbol & Resina \\
\hline Vismia angusta Miq. & Pichirina & CLUSIACEAE & Arbol & $\begin{array}{l}\text { Hoja y } \\
\text { Corteza }\end{array}$ \\
\hline
\end{tabular}

\footnotetext{
* Especie no amazónica.
} 
Cuadro 4: Plantas medicinales exportadas en 1998 y principio de 1999*.

\begin{tabular}{|c|c|c|c|c|}
\hline Nombre científico & $\begin{array}{l}\text { Nombre } \\
\text { vulgar }\end{array}$ & $\begin{array}{c}\text { Parte } \\
\text { utilizada }\end{array}$ & $\begin{array}{l}\text { Cantidad } \\
\text { vendida } \\
\text { en } 1998 \\
\end{array}$ & $\begin{array}{c}\text { Cantidad } \\
\text { vendida hasta } \\
\text { junio } 1999\end{array}$ \\
\hline $\begin{array}{l}\text { Banisteriopsis caapi } \\
\text { (Spruce ex Grise.) }\end{array}$ & Ayahuasca & Corteza & $4990,5 \mathrm{~kg}$ & $50 \mathrm{~kg}$ \\
\hline $\begin{array}{l}\text { Brosimum acutifolium } \\
\text { Huber Subsp. }\end{array}$ & Murure & $\begin{array}{l}\text { Corteza } \\
\text { Resina }\end{array}$ & $25 \mathrm{~kg}$ & $\begin{array}{c}- \\
101\end{array}$ \\
\hline Bursera graveolens (H.B.K.) & Palo Santo & Corteza & - & $40 \mathrm{~kg}$ \\
\hline Copaifera paupera (Herzog) & Copaiba & Resina & 1401 & 21 \\
\hline Croton lechleri Muell. Arg. & $\begin{array}{l}\text { Sangre de } \\
\text { grado }\end{array}$ & Resina & 50607,51 & 316851 \\
\hline Dacryodes peruviana (Loes.) & Copal & Resina & 7,251 & - \\
\hline Diplopterys cabrerana & Chariponga & Hoja & $5 \mathrm{~kg}$ & $9 \mathrm{~kg}$ \\
\hline $\begin{array}{l}\text { Dracontium loretense } \mathrm{K} \text {. } \\
\text { Krause }\end{array}$ & Jergón sacha & Bulbo & $2 \mathrm{~kg}$ & - \\
\hline Ficus insipida (Will.) & Ojé & Resina & 9401 & 7885 \\
\hline Genipa americana L. & Huito & Extracto & - & 41 \\
\hline $\begin{array}{l}\text { Hevea brasiliensis (Will.) } \\
\text { Muell. Arg. }\end{array}$ & Chiringa & Resina & - & 291 \\
\hline $\begin{array}{l}\text { Maytenus macrocarpa } \\
(\mathrm{R} \& \mathrm{P}) \text { Briquet }\end{array}$ & Chuchuhuasi & Corteza & $50 \mathrm{~kg}$ & - \\
\hline Nicotiana tabacum $\mathrm{L}$. & $\begin{array}{l}\text { Mapacho } \\
\text { o Tabaco }\end{array}$ & Hoja & $45,5 \mathrm{~kg}$ & $225 \mathrm{~kg}$ \\
\hline Psychotria viridis $\mathrm{R} \& \mathrm{P}$ & Chacruna & Hoja & $4786,5 \mathrm{~kg}$ & $89 \mathrm{~kg}$ \\
\hline $\begin{array}{l}\text { Trichocereus pachanoi } \\
\text { (Britton \& Rose) }\end{array}$ & San Pedro & Hoja & $21,5 \mathrm{~kg}$ & $110 \mathrm{~kg}$ \\
\hline $\begin{array}{l}\text { Uncaria tomentosa (Will.) } \\
\text { D.C. }\end{array}$ & Uña de gato & Corteza & \multirow[t]{2}{*}{$775 \mathrm{~kg}$} & \multirow[t]{2}{*}{$1175 \mathrm{~kg}$} \\
\hline $\begin{array}{l}\text { Uncaria guianensis (Aubl.) } \\
\text { Gmelin }\end{array}$ & Uña de gato & Corteza & & \\
\hline Virola calophylla Warburg & $\begin{array}{l}\text { Cumala } \\
\text { blanca }\end{array}$ & Resina & 81 & - \\
\hline Vismia angusta Miq. & Pichirina & $\begin{array}{c}\text { Hoja } \\
\text { Corteza }\end{array}$ & $\begin{array}{l}93 \mathrm{~kg} \\
35 \mathrm{~kg}\end{array}$ & - \\
\hline Diversas plantas & $\begin{array}{l}\text { Muestra de } \\
\text { plantas }\end{array}$ & - & $440 \mathrm{~kg}$ & - \\
\hline
\end{tabular}

* Fuente: Dirección Regional de Agricultura de Iquitos. $\square$ Especies más vendidas. 